\title{
Síntese de Redes de Distribuição de Água considerando os sentidos de escoamento desconhecidos
}

\author{
Water Distribution Networks synthesis considering \\ unknown flow directions
}

Gustavo Henrique Batista Cassiolato ${ }^{1}$, Esdras Penêdo de Carvalho ${ }^{1}$ (i), Mauro Antonio da Silva Sá Ravagnani ${ }^{1}$

${ }^{1}$ Universidade Estadual de Maringá - UEM, Maringá, PR, Brasil. E-mails: ghbcassiolato2@uem.br, epcarvalho@uem.br, massravagnani@uem.br

\begin{abstract}
Como citar: Cassiolato, G. H. B., Carvalho, E. P., \& Ravagnani, M. A. S. S. (2022). Síntese de Redes de Distribuição de Água considerando os sentidos de escoamento desconhecidos. Revista de Gestão de Água da América Latina, 19, e3. https://doi.org/10.21168/rega.v19e3
\end{abstract}

Resumo: As Redes de Distribuição de Água (RDA) são responsáveis pelo transporte de água potável em centros urbanos e instalações industriais. Estas redes são formadas por diversos componentes e sua síntese pode ser formulada como um problema de otimização com formulação de Programação Não Linear Inteira Mista (MINLP), envolvendo a minimização do custo de instalação da rede, considerando os comprimentos dos tubos e os sentidos de escoamento conhecidos e um conjunto discreto de diâmetros comerciais. Entretanto, em um projeto real de RDA, os sentidos de escoamento da água são desconhecidos. Neste trabalho, é proposto um modelo de otimização para a síntese de RDA considerando os sentidos de escoamento desconhecidos nos tubos, usando técnicas de programação disjuntiva, seguindo uma abordagem determinística. Três estudos de caso da literatura são explorados para testar a aplicabilidade do modelo e técnicas de otimização global são usadas na sua resolução.

Palavras-chave: Redes de Distribuição de Água; Programação Matemática; Sentido de Escoamento Desconhecido; Custos de Instalação.

\begin{abstract}
Water Distribution Networks (WDN) are systems responsible for potable water transportation in urban centers and industrial installations and are formed by several components. WDN synthesis can be formulated as an optimization problem with Mixed Integer Non-Linear Programming (MINLP) formulation, involving the minimization of the network installation cost, considering the tubes length and known flow directions and a discrete set of available diameters. However, in real WDN flow directions are unknown. In the present paper it is proposed an optimization model for WDN synthesis considering unknown flow directions, using disjunctive programming techniques and a deterministic approach. Three case studies are explored to test the model applicability and global optimization techniques are used in the problem solution.
\end{abstract}

Keywords: Water Distribution Networks; Mathematical Programming; Unknown Flow Directions; Installation Costs.

\section{INTRODUÇÃO}

A superfície terrestre, em sua maioria, é composta pelo principal recurso natural existente para o desenvolvimento de seres vivos, a água. A maior parte dessa água é contemplada pela água salgada. A água doce é usada pelos seres humanos, sendo originária em lagos, rios, açudes e entre outros. Para que haja o fornecimento de água aos consumidores é necessário um sistema de abastecimento.

No desenvolvimento de grandes e pequenas regiões, os sistemas de abastecimento de água são fundamentais e fazem parte das necessidades básicas de qualquer população. Estes sistemas são compostos principalmente pela captação de água, por uma estação de tratamento e uma rede de distribuição. Os custos de capital decorrentes da aquisição e instalação da rede de distribuição são os mais expressivos no sistema de abastecimento, dependendo da população a ser atendida.

As Redes de Distribuição de Água (RDA) são responsáveis por transportar a água tratada para todos os pontos de demanda e devem proporcionar o fornecimento de forma adequada e eficiente,

Recebido: Agosto 10, 2021. Revisado: Dezembro 16, 2021. Aceito: Dezembro 27, 2021. 
reduzindo ao máximo os desperdícios ao longo de sua operação. As RDA são formadas por um conjunto de equipamentos, contendo essencialmente nós de demanda de água, tubos caracterizados por seus nós iniciais e finais, reservatórios de água e bombas hidráulicas. Algumas tubulações podem constituir loops fechados entre os nós de demanda e a água pode ser fornecida por gravidade ou usando um sistema de bombeamento.

O projeto de uma RDA é um problema complexo que pode ser formulado como um problema de otimização. A maioria dos trabalhos existentes na literatura busca minimizar o custo de instalação da rede, sendo os diâmetros dos tubos as variáveis discretas de otimização, selecionados de acordo com um conjunto de diâmetros comerciais, enquanto os comprimentos dos tubos e os sentidos de escoamento da água são previamente definidos. As restrições do problema são o balanço de massa em cada nó, a diferença de pressão entre dois nós, considerando a existência de loops e a velocidade da água em cada tubo e a queda de pressão devem obedecer a limites operacionais, formando um sistema de equações não lineares, que pode ter várias soluções. Na literatura, diferentes abordagens determinísticas e estocásticas têm sido usadas para resolver o problema (Mala-Jetmarova et al., 2018).

Quando uma nova RDA é projetada em setores urbanos ou em processos industriais, os sentidos de escoamento da água nos tubos não são conhecidos e a solução do problema de otimização pode se tornar ainda mais difícil de ser obtida. No presente trabalho, para representar a RDA propõe-se um modelo de otimização com uma formulação de Programação Não Linear Inteira Mista (MINLP), considerando os diâmetros dos tubos e os sentidos de escoamento como variáveis de otimização. 0 modelo proposto considera uma abordagem determinística com a utilização de programação disjuntiva, com o uso de restrições algébricas e expressões lógicas simbólicas. Algumas transformações logarítmicas são introduzidas a fim de tornar o modelo mais robusto e evitar problemas associados à não linearidade do sistema. 0 ambiente GAMS (General Algebraic Modeling System) é usado para resolver o problema, sem o uso de simuladores hidráulicos. Estudos de caso da literatura são utilizados para testar a aplicação do modelo desenvolvido e técnicas de otimização global são usadas na sua resolução.

\section{REVISÃO DA LITERATURA}

Na literatura os trabalhos sobre o projeto ótimo de RDA consideram distintas abordagens. Problemas de otimização com formulação de Programação Linear (LP), Programação Não Linear (NLP), Programação Linear Inteira Mista (MILP) e Programação Não Linear Inteira Mista têm sido propostos, envolvendo técnicas determinísticas ou estocásticas para a sua solução. As formulações MINLP são mais representativas para problemas reais.

Alperovits \& Shamir (1977) resolveram um problema LP para obter uma distribuição viável e depois realizaram uma busca no espaço das variáveis, com base no gradiente da função objetivo. Este método foi expandido em Goulter et al. (1986), contemplando a importância da escolha do caminho de perda de carga no problema de otimização, em Kessler \& Shamir (1989), apresentando uma análise do método e uma formulação usando a teoria de grafos e, em Eiger et al. (1994), abordando um problema semi-infinito, resolvido globalmente por um algoritmo Branch and Bound, usando teoria da dualidade. Hansen et al. (1991) propuseram um algoritmo sucessivo com uma abordagem de busca local e Sarbu (1997) obteve resultados interessantes em novas RDA, bem como para a extensão de RDA existentes.

Morsi et al. (2012) propuseram um modelo de otimização de RDA do tipo MILP e o método Branch and Bound foi usado para a resolução do problema. Os primeiros autores que consideraram as formulações NLP para o projeto de RDA foram Shamir \& Howard (1968). 0 método de NewtonRaphson foi usado para resolver o sistema de equações. Watanatada (1973) também contribuiu com uma formulação NLP para resolver o problema de projeto de RDA, usando restrições de conservação de massa e energia e limites físicos. As restrições de desigualdade foram limitadas por uma transformação de Box e as de igualdade foram resolvidas usando o método de Haarhoff e Buys.

Modelos MINLP e métodos determinísticos para a síntese da RDA foram propostos nos trabalhos de Bragalli et al. $(2006,2008,2012)$. Neste último trabalho os autores propuseram um relaxamento nas variáveis e resolveram o problema com o solver BONMIN, no ambiente AMPL. D'Ambrosio et al. (2015) desenvolveram um modelo com formulação MINLP para descrever a dinâmica da água em tubulações, usando ramificações espaciais e relaxamentos lineares. Cassiolato et al. (2019) propuseram uma formulação disjuntiva do tipo Big-M para a síntese de RDA e o solver SBB foi usado, no ambiente GAMS (General Algebraic Modeling System). Cassiolato et al. (2021) também 
apresentaram um modelo do tipo MINLP com uma reformulação Convex Hull e os solvers SBB e BARON foram aplicados, em GAMS.

A maioria dos trabalhos publicados, no entanto, usa abordagens não determinísticas para resolver o problema. As principais meta-heurísticas utilizadas são Genetic Algorithms (GA), nos trabalhos de "Savic, Walters (1997), Reca \& Martínez (2006), Kadu et al. (2008), Bi et al. (2015) e Reca et al. (2017); Artificial Immune Systems (AIS), utilizada por Eryiğit (2015); Harmony Search (HS), nos trabalhos de Geem (2006, 2009); Particle Swarm Optimization (PSO), nos trabalhos de Suribabu \& Neelakantan (2006), Ezzeldin et al. (2014), Rao et al. (2017), Surco et al. (2018a, 2018b, 2019, 2021); Differential Evolution (DE), nos trabalhos de Vasan \& Simonovic (2010), Suribabu (2010) e Suribabu \& Neelakanta (2014); Gravitational Search Algorithm (GSA), no trabalho de Fallah et al. (2021); Evolutionary Algorithm (EA), em Bolognesi et al. (2010), Avila-Melgar et al. (2017) e Palod et al. (2020); Simple Benchmarking Algorithm (SBA) em Shende \&Chau (2019) e Whale Optimization Algorithm (WOA), em Ezzeldin \& Djebedjian (2020).

Assim, a maioria das contribuições da literatura para resolver o problema de projeto de RDA usa abordagens não determinísticas, com os sentidos de escoamento da água conhecidos nos tubos e um software auxiliar para solucionar as equações hidráulicas pertencentes ao problema. No presente trabalho apresenta-se um modelo de otimização com a formulação MINLP, considerando os sentidos de escoamento da água desconhecidos e sem a necessidade de utilizar software adicional.

\section{MODELO DE OTIMIZAÇÃo}

O modelo proposto para projeto ótimo de RDA consiste na determinação dos diâmetros dos tubos, formulado como um problema de otimização com formulação MINLP. Os sentidos de escoamento da água são considerados desconhecidos nos tubos e são caracterizados por variáveis binárias, de acordo com a proposição de disjunções. Define-se como $\mathcal{N}$ o conjunto de índices $(i, j)$ para os nós da RDA e denota-se como $i-j$ o tubo cujo escoamento da água está indo do nó $i$ para o nó $j$. Considere-se $\mathcal{E}_{i, j} \subset \mathcal{N}$ o subconjunto de índices para os nós da RDA em que existe uma tubulação ligando $i-j$.

Sejam $d_{i}$ a demanda de água para o nó $i, q_{i, j}$ a vazão volumétrica no tubo $i-j, q_{i, j}^{1}$ é igual a $q_{i, j}$ se a água flui do nó $i$ para o nó $j$ e, em caso contrário, vale zero. A vazão $q_{i, j}^{2}$ é igual a $q_{i, j}$ se a água flui do nó $j$ para o nó $i$, ou, em caso contrário, vale zero. 0 balanço de massa em cada nó de demanda é:

$$
\sum_{j \in \mathcal{E}_{j, i}}\left(q_{j, i}^{1}-q_{j, i}^{2}\right)-\sum_{j \in \mathcal{E}_{i, j}}\left(q_{i, j}^{1}-q_{i, j}^{2}\right)=d_{i}, \forall i \in \mathcal{N}
$$

A grande dificuldade nesse tipo de balanço é que nos laços existentes nas RDA não se sabe, a priori, em que sentido está escoando a água. Por isso, faz-se necessário expressar a vazão como a diferença de dois termos. Define-se, assim, o conjunto $\mathcal{E}_{i, j}$. Faz-se $q_{i, j}^{1}>0$ se a água escoa do nó $i$ para o nó $j$ e $q_{i, j}^{2}>0$ se a água escoa do nó $j$ para o nó $i$.

Sejam $P_{i}$ a pressão no nó $i$ e $h_{i}$ a elevação do nó $i$. Considere-se $E p_{i, j}$ a altura manométrica, ou elevação de pressão, gerada por uma bomba hidráulica no tubo $i-j$ e $E p_{i, j}^{1}$ igual a $E p_{i, j}$ se a água flui do nó $i$ para o nó $j$. Caso contrário, vale zero, e $E p_{i, j}^{2}$ é igual a $E p_{i, j}$, se a água flui do nó $j$ para o nó $i$. Caso contrário, vale zero. Define-se $\Delta P_{i, j}$ como sendo a queda de pressão no tubo $i-j$ e $\Delta P_{i, j}^{1}$ igual a $\Delta P_{i, j}$ se a água flui do nó $i$ para o nó $j$. Caso contrário, vale zero. Se a água flui do nó $j$ para o nó $i$, $\Delta P_{i, j}^{2}$ é igual a $\Delta P_{i, j}$ e, em caso contrário, vale zero. A diferença de pressão entre dois nós pode ser escrita da seguinte maneira:

$$
P_{i}+h_{i}+E p_{i, j}^{1}-E p_{i, j}^{2}=P_{j}+h_{j}+\Delta P_{i, j}^{1}-\Delta P_{i, j}^{2}, \forall i, j \in \mathcal{E}_{i, j}
$$

em que $P_{i}^{\text {min }} \leq P_{i}$, sendo $P_{i}^{\text {min }}$ a pressão mínima permitida no nó $i$, para todo $i \in \mathcal{N}$.

$L_{i, j}$ é o comprimento do tubo ligando os nós $i-j, x_{i, j}$ é o diâmetro do tubo e $v_{i, j}$ a velocidade da água no trecho $i-j$. A equação mais utilizada na literatura para o cálculo da queda de pressão quando a água é o fluido em escoamento é a de Hazen-Williams. Seja $C_{i, j}$ o coeficiente de rugosidade de HazenWilliams no trecho $i-j$, que é um valor adimensional e dependente do material e do tempo de uso. Essa equação é dada por: 
$\Delta P_{i, j}=\frac{\alpha L_{i, j} q_{i, j}^{\beta}}{C_{i, j}^{\beta} x_{i, j}^{\gamma}}, \forall i, j \in \mathcal{E}_{i, j}$

em que $\alpha$ é um fator de conversão numérica associado ao sistema de unidades utilizado na equação e $\beta=1 / 0,54$ e $\gamma=2,63 / 0,54$ são coeficientes fixos. A equação de Hazen-Williams é sensível a essas constantes, ou seja, pequenas mudanças nos valores podem alterar significativamente a solução do problema.

A velocidade da água em cada trecho $i-j$ é dada por:

$v_{i, j}=\frac{4 q_{i, j}}{\pi x_{i, j}^{2}}, \forall i, j \in \mathcal{E}_{i, j}$

As Equações 3 e 4 são não lineares. Para evitar problemas afetos à não linearidade dessas equações, faz-se uso de logaritmos, transformando as equações não lineares em lineares:

$$
\begin{aligned}
& \ln \Delta P_{i, j}=\ln \left(\alpha L_{i, j}\right)+\beta \ln q_{i, j}-\ln \left(C_{i, j}^{\beta} x_{i, j}^{\gamma}\right), \forall i, j \in \mathcal{E}_{i, j} \\
& \ln v_{i, j}=\ln q_{i, j}-\ln \left(\frac{\pi}{4} x_{i, j}^{2}\right), \forall i, j \in \mathcal{E}_{i, j}
\end{aligned}
$$

Definindo as variáveis $\bar{v}_{i, j}=\ln v_{i, j}, \bar{q}_{i, j}=\ln q_{i, j}$ e $\Delta \bar{P}_{i, j}=\ln \Delta P_{i, j}$, para todo $i, j \in \mathcal{E}_{i, j}$, e utilizando-as nas Equações 5 e 6, todos os termos nessas equações serão lineares, uma vez que os diâmetros, os comprimentos e os coeficientes de rugosidade de Hazen-Williams dos tubos são valores conhecidos. Assim, obtém-se as seguintes equações:

$$
\begin{aligned}
& \Delta \bar{P}_{i, j}=\ln \left(\alpha L_{i, j}\right)+\beta \bar{q}_{i, j}-\ln \left(C_{i, j}^{\beta} x_{i, j}^{\gamma}\right), \forall i, j \in \mathcal{E}_{i, j} \\
& \bar{v}_{i, j}=\bar{q}_{i, j}-\ln \left(\frac{\pi}{4} x_{i, j}^{2}\right), \forall i, j \in \mathcal{E}_{i, j}
\end{aligned}
$$

As variáveis originais $v, q$ e $\Delta P$ podem ser encontradas pela exponenciação dos valores correspondentes de $\bar{v}, \bar{q}$ e $\Delta \bar{P}$ :

$$
\begin{gathered}
e^{\bar{v}_{i, j}}=v_{i, j}, \forall i, j \in \mathcal{E}_{i, j} \\
e^{\bar{q}_{i, j}}=q_{i, j}, \forall i, j \in \mathcal{E}_{i, j} \\
e^{\Delta \bar{P}_{i, j}}=\Delta P_{i, j}, \forall i, j \in \mathcal{E}_{i, j}
\end{gathered}
$$

Seja o custo por comprimento do tubo $i-j$ com diâmetro $x_{i, j}$ dado por $\operatorname{Custo}\left(x_{i, j}\right)$ e $C_{T}$ o custo total de instalação da rede, a função objetivo a ser minimizada é dada por:

$$
C_{T}=\sum_{i, j \in \mathcal{E}_{i, j}} L_{i, j} \operatorname{Custo}\left(x_{i, j}\right)
$$

Sejam $D_{k}$ o diâmetro comercial disponível, $\operatorname{Custo}\left(D_{k}\right)$ o custo por comprimento do tubo com diâmetro $D_{k}$ e $R_{k}$ a rugosidade do tubo com diâmetro $D_{k}$, para todo $k \in \mathcal{D}$, em que $\mathcal{D}$ é o conjunto de índices para os diâmetros disponíveis para a RDA.

Para evitar aproximações ou arredondamentos, devido à existência de variáveis discretas, uma reformulação no modelo é proposta, usando programação disjuntiva, que é uma técnica que relaciona variáveis discretas com conceitos de lógica matemática (Balas, 2018).

Dado um par $i, j \in \mathcal{E}_{i, j}$, sejam $Y_{i, j, k}$ a variável booleana associada ao trecho $i-j$ da tubulação com diâmetro $D_{k}$, para todo $k \in \mathcal{D}$. Esta variável booleana é verdadeira se no trecho $i-j$ o diâmetro $D_{k}$ for 
selecionado e falsa, caso contrário. Define-se $\lambda_{i, j}$ o custo do trecho $i-j$ e $\sigma_{i, j}$ a rugosidade do tubo nesse trecho. Desta forma, a disjunção exclusiva que caracteriza a escolha do diâmetro de cada tubo é escrita como sendo:

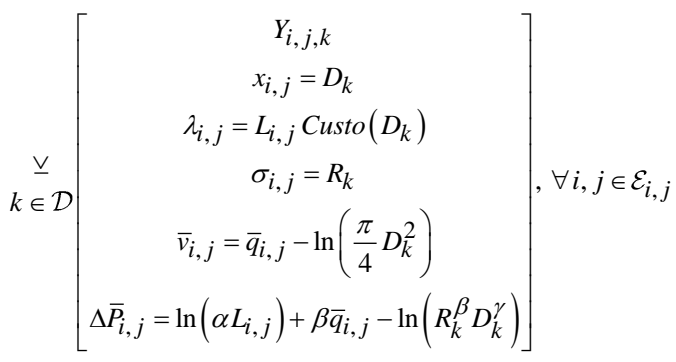

Esta disjunção pode ser reescrita usando uma reformulação convexa para variáveis discretas (Grossmann \& Lee, 2003). Seja $y_{i, j, k}$ a variável binária associada ao trecho $i-j$ com diâmetro $D_{k}$, para todo $k \in \mathcal{D}$, que vale 1 se no trecho $i-j$ o diâmetro $D_{k}$ for selecionado e 0 , caso contrário. Desta forma, têm-se as equações:

$$
\begin{aligned}
& x_{i, j}=\sum_{k \in \mathcal{D}} D_{k} y_{i, j, k}, \forall i, j \in \mathcal{E}_{i, j} \\
& \lambda_{i, j}=\sum_{k \in \mathcal{D}} L_{i, j} \operatorname{Custo}\left(D_{k}\right) y_{i, j, k}, \forall i, j \in \mathcal{E}_{i, j} \\
& \sigma_{i, j}=\sum_{k \in \mathcal{D}} R_{k} y_{i, j, k}, \forall i, j \in \mathcal{E}_{i, j} \\
& \bar{v}_{i, j}=\bar{q}_{i, j}-\sum_{k \in \mathcal{D}} \ln \left(\frac{\pi}{4} D_{k}^{2}\right) y_{i, j, k}, \forall i, j \in \mathcal{E}_{i, j} \\
& \Delta \bar{P}_{i, j}=\ln \left(\alpha L_{i, j}\right)+\beta \bar{q}_{i, j}-\sum_{k \in \mathcal{D}} \ln \left(R_{k}^{\beta} D_{k}^{\gamma}\right) y_{i, j, k}, \forall i, j \in \mathcal{E}_{i, j} \\
& \sum_{k \in \mathcal{D}} y_{i, j, k}=1, \forall i, j \in \mathcal{E}_{i, j}
\end{aligned}
$$

A Equação 19 indica que para cada trecho $i-j$ apenas um diâmetro pode ser escolhido. Como o custo de cada tubo depende do diâmetro, então a nova função objetivo, que representa o custo total de instalação da rede, é dada por:

$$
C_{T}=\sum_{i, j \in \mathcal{E}_{i, j}} \lambda_{i, j}
$$

Sejam $v_{i, j}^{1}$ igual a $v_{i, j}$ se a água flui do nó $i$ para o nó $j$ e zero, em caso contrário, e $v_{i, j}^{2}$ igual a $v_{i, j}$ se a água flui do nó $j$ para o nó $i$ e zero em caso contrário. Para que $q_{i, j}^{1}, q_{i, j}^{2}, \Delta P_{i, j}^{1}, \Delta P_{i, j}^{2}, E p_{i, j}^{1}$, $E p_{i, j}^{2}, v_{i, j}^{1}$ e $v_{i, j}^{2}$ sejam diferentes de zero, existe a necessidade de impor a elas limites inferior e superior. Define-se os limites inferiores como sendo $q_{i, j}^{\min }, \Delta P_{i, j}^{\min }, E p_{i, j}^{\min }$ e $v_{i, j}^{\min }$, para todo $i, j \in \mathcal{E}_{i, j}$. Analogamente, $q_{i, j}^{\max }, \Delta P_{i, j}^{\max }, E p_{i, j}^{\max }$ e $v_{i, j}^{\max }$ são os limites superiores.

Dado um par $i, j \in \mathcal{E}_{i, j}$, sejam $w_{i, j}^{1}$ a variável booleana que é verdadeira se a água escoa do nó $i$ para o nó $j$ e falsa, caso contrário, e $w_{i, j}^{2}$ a variável booleana que é verdadeira se a água escoa do nó $j$ para o nó $i$ e falsa, caso contrário. Desta forma, a disjunção exclusiva que caracteriza a escolha do sentido de escoamento de cada tubo é escrita por: 


$$
\left.\left[\begin{array}{c}
W_{i, j}^{1} \\
v_{i, j}=v_{i, j}^{1} \\
q_{i, j}=q_{i, j}^{1} \\
\Delta P_{i, j}=\Delta P_{i, j}^{1} \\
E p_{i, j}=E p_{i, j}^{1} \\
v_{i, j}^{\min } \leq v_{i, j} \leq v_{i, j}^{\max } \\
q_{i, j}^{\min } \leq q_{i, j} \leq q_{i, j}^{\max } \\
\Delta P_{i, j}^{\min } \leq \Delta P_{i, j} \leq \Delta P_{i, j}^{\max } \\
E p_{i, j}^{\min } \leq E p_{i, j} \leq E p_{i, j}^{\max }
\end{array}\right] \underline{W_{i, j}^{2}} \begin{array}{c}
v_{i, j}=v_{i, j}^{2} \\
q_{i, j}=q_{i, j}^{2} \\
\Delta P_{i, j}=\Delta P_{i, j}^{2} \\
E p_{i, j}=E p_{i, j}^{2} \\
v_{i, j}^{\min } \leq v_{i, j} \leq v_{i, j}^{\max } \\
q_{i, j}^{\min } \leq q_{i, j} \leq q_{i, j}^{\max } \\
\Delta P_{i, j}^{\min } \leq \Delta P_{i, j} \leq \Delta P_{i, j}^{\max } \\
E p_{i, j}^{\min } \leq E p_{i, j} \leq E p_{i, j}^{\max }
\end{array}\right], \forall i, j \in \varepsilon_{i, j}
$$

Esta disjunção pode ser escrita usando uma reformulação convexa para variáveis contínuas limitadas. Seja $w_{i, j}^{1}$ a variável binária que vale 1 se a água escoa do nó $i$ para o nó $j$ e 0 , caso contrário, e $w_{i, j}^{2}$ a variável binária que vale 1 se a água escoa do nó $j$ para o nó $i$ e 0 , caso contrário. Desta forma, têm-se as equações:

$$
\begin{aligned}
& v_{i, j}=v_{i, j}^{1}+v_{i, j}^{2}, \forall i, j \in \mathcal{E}_{i, j} \\
& v_{i, j}^{\min } w_{i, j}^{1} \leq v_{i, j}^{1} \leq v_{i, j}^{\max } w_{i, j}^{1}, \forall i, j \in \mathcal{E}_{i, j} \\
& v_{i, j}^{m i n} w_{i, j}^{2} \leq v_{i, j}^{2} \leq v_{i, j}^{\max } w_{i, j}^{2}, \forall i, j \in \mathcal{E}_{i, j} \\
& q_{i, j}=q_{i, j}^{1}+q_{i, j}^{2}, \forall i, j \in \mathcal{E}_{i, j} \\
& q_{i, j}^{\min } w_{i, j}^{1} \leq q_{i, j}^{1} \leq q_{i, j}^{\max } w_{i, j}^{1}, \forall i, j \in \mathcal{E}_{i, j} \\
& q_{i, j}^{m i n} w_{i, j}^{2} \leq q_{i, j}^{2} \leq q_{i, j}^{\max } w_{i, j}^{2}, \forall i, j \in \mathcal{E}_{i, j} \\
& \Delta P_{i, j}=\Delta P_{i, j}^{1}+\Delta P_{i, j}^{2}, \forall i, j \in \mathcal{E}_{i, j} \\
& \Delta P_{i, j}^{\min } w_{i, j}^{1} \leq \Delta P_{i, j}^{1} \leq \Delta P_{i, j}^{\max } w_{i, j}^{1}, \forall i, j \in \mathcal{E}_{i, j} \\
& \Delta P_{i, j}^{m i n} w_{i, j}^{2} \leq \Delta P_{i, j}^{2} \leq \Delta P_{i, j}^{m a x} w_{i, j}^{2}, \forall i, j \in \mathcal{E}_{i, j} \\
& E p_{i, j}=E p_{i, j}^{1}+E p_{i, j}^{2}, \forall i, j \in \mathcal{E}_{i, j} \\
& E p_{i, j}^{\min } w_{i, j}^{1} \leq E p_{i, j}^{1} \leq E p_{i, j}^{\max } w_{i, j}^{1}, \forall i, j \in \mathcal{E}_{i, j} \\
& E p_{i, j}^{\min } w_{i, j}^{2} \leq E p_{i, j}^{2} \leq E p_{i, j}^{\max } w_{i, j}^{2}, \forall i, j \in \mathcal{E}_{i, j} \\
& w_{i, j}^{1}+w_{i, j}^{2}=1, \forall i, j \in \mathcal{E}_{i, j}
\end{aligned}
$$

Portanto, o modelo de otimização para a síntese de RDA considerando os sentidos de escoamento desconhecidos nos tubos é dado por:

$\min \sum_{i, j \in \mathcal{E}_{i, j}} \lambda_{i, j}$ 


$$
\begin{aligned}
& \text { s. a } \sum_{j \in \mathcal{E}_{j, i}}\left(q_{j, i}^{1}-q_{j, i}^{2}\right)-\sum_{j \in \mathcal{E}_{i, j}}\left(q_{i, j}^{1}-q_{i, j}^{2}\right)=d_{i}, \forall i \in \mathcal{N} \\
& P_{i}+h_{i}+E p_{i, j}^{1}-E p_{i, j}^{2}=P_{j}+h_{j}+\Delta P_{i, j}^{1}-\Delta P_{i, j}^{2}, \forall i, j \in \mathcal{E}_{i, j} \\
& x_{i, j}=\sum_{k \in \mathcal{D}} D_{k} y_{i, j, k}, \forall i, j \in \mathcal{E}_{i, j} \\
& \lambda_{i, j}=\sum_{k \in \mathcal{D}} L_{i, j} \operatorname{Custo}\left(D_{k}\right) y_{i, j, k}, \forall i, j \in \mathcal{E}_{i, j} \\
& \sigma_{i, j}=\sum_{k \in \mathcal{D}} R_{k} y_{i, j, k}, \forall i, j \in \mathcal{E}_{i, j} \\
& \bar{v}_{i, j}=\bar{q}_{i, j}-\sum_{k \in \mathcal{D}} \ln \left(\frac{\pi}{4} D_{k}^{2}\right) y_{i, j, k}, \forall i, j \in \mathcal{E}_{i, j} \\
& \Delta \bar{P}_{i, j}=\ln \left(\alpha L_{i, j}\right)+\beta \bar{q}_{i, j}-\sum_{k \in \mathcal{D}} \ln \left(R_{k}^{\beta} D_{k}^{\gamma}\right) y_{i, j, k}, \forall i, j \in \mathcal{E}_{i, j} \\
& \sum_{k \in \mathcal{D}} y_{i, j, k}=1, \forall i, j \in \mathcal{E}_{i, j} \\
& e^{\bar{v}_{i, j}}=v_{i, j}, \forall i, j \in \mathcal{E}_{i, j} \\
& e^{\bar{q}_{i, j}}=q_{i, j}, \forall i, j \in \mathcal{E}_{i, j} \\
& e^{\Delta \bar{P}_{i, j}}=\Delta P_{i, j}, \forall i, j \in \mathcal{E}_{i, j} \\
& P_{i}^{\min } \leq P_{i}, \forall i \in \mathcal{N} \\
& v_{i, j}=v_{i, j}^{1}+v_{i, j}^{2}, \forall i, j \in \mathcal{E}_{i, j} \\
& v_{i, j}^{\min } w_{i, j}^{1} \leq v_{i, j}^{1} \leq v_{i, j}^{\max } w_{i, j}^{1}, \forall i, j \in \mathcal{E}_{i, j} \\
& v_{i, j}^{\min } w_{i, j}^{2} \leq v_{i, j}^{2} \leq v_{i, j}^{\max } w_{i, j}^{2}, \forall i, j \in \mathcal{E}_{i, j} \\
& q_{i, j}=q_{i, j}^{1}+q_{i, j}^{2}, \forall i, j \in \mathcal{E}_{i, j} \\
& q_{i, j}^{\min } w_{i, j}^{1} \leq q_{i, j}^{1} \leq q_{i, j}^{\max } w_{i, j}^{1}, \forall i, j \in \mathcal{E}_{i, j} \\
& q_{i, j}^{\min } w_{i, j}^{2} \leq q_{i, j}^{2} \leq q_{i, j}^{\max } w_{i, j}^{2}, \forall i, j \in \mathcal{E}_{i, j} \\
& \Delta P_{i, j}=\Delta P_{i, j}^{1}+\Delta P_{i, j}^{2}, \forall i, j \in \mathcal{E}_{i, j} \\
& \Delta P_{i, j}^{\min } w_{i, j}^{1} \leq \Delta P_{i, j}^{1} \leq \Delta P_{i, j}^{\max } w_{i, j}^{1}, \forall i, j \in \mathcal{E}_{i, j} \\
& \Delta P_{i, j}^{\min } w_{i, j}^{2} \leq \Delta P_{i, j}^{2} \leq \Delta P_{i, j}^{\max } w_{i, j}^{2}, \forall i, j \in \mathcal{E}_{i, j} \\
& E p_{i, j}=E p_{i, j}^{1}+E p_{i, j}^{2}, \forall i, j \in \mathcal{E}_{i, j}
\end{aligned}
$$




$$
\begin{aligned}
& E p_{i, j}^{\min } w_{i, j}^{1} \leq E p_{i, j}^{1} \leq E p_{i, j}^{\max } w_{i, j}^{1}, \forall i, j \in \mathcal{E}_{i, j} \\
& E p_{i, j}^{\min } w_{i, j}^{2} \leq E p_{i, j}^{2} \leq E p_{i, j}^{\max } w_{i, j}^{2}, \forall i, j \in \mathcal{E}_{i, j} \\
& w_{i, j}^{1}+w_{i, j}^{2}=1, \forall i, j \in \mathcal{E}_{i, j}
\end{aligned}
$$

\section{ESTUDOS DE CASO}

Para a aplicação da abordagem desenvolvida, três estudos de caso foram escolhidos da literatura. 0 modelo foi implementado no ambiente GAMS e as soluções dos problemas foram obtidas usando técnicas de otimização global. Em todos os casos analisados, os sentidos de escoamento foram considerados variáveis de otimização, juntamente com os diâmetros dos tubos e com o custo total de instalação da rede. Quando os sentidos de escoamento são considerados desconhecidos, invariavelmente, ocorre um acréscimo no tempo de processamento. Porém, como demonstrado nos casos estudados, esse acréscimo não é significativo para a obtenção dos resultados, como pode ser observado nos casos a seguir.

\subsection{Estudo de Caso 1}

Esse estudo de caso refere-se ao dimensionamento de parte da rede de distribuição de água do bairro do Bessa na cidade de João Pessoa, Paraíba, projetada pela Companhia de Águas e Esgoto da Paraíba (CAGEPA) em 1982. A Figura 1 representa sua topologia, contendo 1 reservatório, 7 tubos interligando 7 nós de consumo e 1 loop. A alimentação dessa rede é feita por gravidade e o reservatório é representado pelo primeiro nó. As características dos nós e os comprimentos dos tubos são apresentados na Tabela 1. Para a rede Bessa são disponibilizados 10 diâmetros comerciais, totalizando $10^{7}$ possíveis soluções para o problema (Tabela 2). A velocidade da água deve ser limitada entre $0,3 \mathrm{~m} / \mathrm{s}$ e $3 \mathrm{~m} / \mathrm{s}$, e a pressão mínima é de $25 \mathrm{~m}$ para todos os nós. Os parâmetros da equação de HazenWilliams são $\alpha=10,667, \beta=1,852$ e $\gamma=4,871$.

O problema foi resolvido com o solver de otimização global BARON. As velocidades da água, os sentidos de escoamento encontrados para os tubos, as pressões calculadas para os nós e uma comparação com os resultados encontrados na literatura são apresentados na Tabela 3. Como pode ser visto, o melhor valor encontrado foi o custo de $\$ 126.806 .220$, que corresponde ao ótimo global. 0 autor citado na comparação dos resultados usou um software adicional para o cálculo das variáveis hidráulicas e considerou os sentidos de escoamento conhecidos.



Figura 1 - Rede Bessa

Tabela 1 - Características dos nós e dos tubos para a rede Bessa

\begin{tabular}{c|c|c|c|c}
\hline \multicolumn{1}{c}{ Nó } & Elevação (m) Demanda (L/s) & 1 & Comprimento (m) \\
\hline 1 & 54,0 & $-420,43$ & 2 & 2540 \\
\hline 2 & 5,0 & 0 & 3 & 150 \\
\hline 3 & 5,0 & 43,44 & 4 & 1140 \\
\hline 4 & 4,0 & 40,29 & 5 & 1430 \\
\hline 5 & 4,5 & 208,6 & 6 & 1710 \\
\hline 6 & 4,5 & 47,78 & 7 & 1410 \\
\hline 7 & 4,5 & 80,32 & & \\
\hline
\end{tabular}


Tabela 2 - Dados dos diâmetros disponíveis para a rede Bessa

\begin{tabular}{c|c|c|c|c|c}
\hline $\begin{array}{c}\text { Diâmetro } \\
(\mathbf{m})\end{array}$ & $\begin{array}{c}\text { Custo } \\
\mathbf{( \$ / m )}\end{array}$ & $\begin{array}{c}\text { Coeficiente } \\
\text { de rugosidade }\end{array}$ & $\begin{array}{c}\text { Diâmetro } \\
\mathbf{( m )}\end{array}$ & $\begin{array}{c}\text { Custo } \\
\text { (\$/m) }\end{array}$ & $\begin{array}{c}\text { Coeficiente } \\
\text { de rugosidade }\end{array}$ \\
\hline 0,10 & 1.629 & 145 & 0,35 & 11.012 & 130 \\
\hline 0,15 & 4.054 & 145 & 0,40 & 12.397 & 130 \\
\hline 0,20 & 5.769 & 145 & 0,45 & 15.501 & 130 \\
\hline 0,25 & 7.718 & 145 & 0,50 & 17.696 & 130 \\
\hline 0,30 & 9.237 & 130 & 0,60 & 23.132 & 130 \\
\hline
\end{tabular}

Tabela 3 - Variáveis hidráulicas, custos e diâmetros calculados para a rede Bessa

\begin{tabular}{|c|c|c|c|c|c|c|c|}
\hline Trecho & $\begin{array}{l}\text { Sentido de } \\
\text { escoam. }\end{array}$ & $\begin{array}{c}\text { Diâmetro (m) } \\
\text { Carvalho } \\
\text { (2007) }\end{array}$ & $\begin{array}{c}\text { Diâmetro (m) } \\
\text { Presente } \\
\text { trabalho }\end{array}$ & $\begin{array}{c}\text { Velocidade } \\
\text { (m/s) } \\
\text { Presente } \\
\text { trabalho } \\
\end{array}$ & Nó & $\begin{array}{c}\text { Pressão (m) } \\
\text { Carvalho } \\
\text { (2007) }\end{array}$ & $\begin{array}{c}\text { Pressão (m) } \\
\text { Presente } \\
\text { trabalho }\end{array}$ \\
\hline 1 & $1-2$ & 0,6 & 0,6 & 1,49 & 1 & \multicolumn{2}{|c|}{ Reservatório } \\
\hline 2 & $2-3$ & 0,5 & 0,45 & 1,82 & 2 & 41,03 & 41,02 \\
\hline 3 & $2-6$ & 0,35 & 0,35 & 1,37 & 3 & 39,69 & 38,8 \\
\hline 4 & $3-4$ & 0,45 & 0,45 & 1,54 & 4 & 35,33 & 34,45 \\
\hline 5 & $4-5$ & 0,4 & 0,4 & 1,63 & 5 & 26,26 & 25,4 \\
\hline 6 & $7-5$ & 0,1 & 0,1 & 0,48 & 6 & 36,39 & 36,36 \\
\hline 7 & $6-7$ & 0,3 & 0,3 & 1,19 & 7 & 29,79 & 29,69 \\
\hline Custo (\$) & & 127.574 .470 & 126.806 .220 & & & & \\
\hline
\end{tabular}

\subsection{Estudo de Caso 2}

Esse estudo de caso foi proposto originalmente por Alperovits \& Shamir (1977) e é conhecido como Two loop. A Figura 2 representa sua topologia, contendo 1 reservatório, 8 tubos interligando 7 nós de consumo e 2 loops. A alimentação dessa rede é feita por gravidade e o reservatório é representado pelo primeiro nó. Todos os tubos possuem $1.000 \mathrm{~m}$ de comprimento e as características dos nós são apresentadas na Tabela 4. Para a rede Two loop são disponibilizados 14 diâmetros comerciais, totalizando $14^{8}$ possíveis soluções para o problema (Tabela 5), em que o coeficiente de rugosidade é 130 para todos os diâmetros. A velocidade da água deve ser limitada entre $0,3 \mathrm{~m} / \mathrm{s}$ e $3 \mathrm{~m} / \mathrm{s}$, e a pressão mínima é de $30 \mathrm{~m}$ para todos os nós. Os parâmetros da equação de Hazen-Williams são $\alpha=10,667, \beta=1,852$ e $\gamma=4,871$.

O problema foi resolvido com o solver de otimização global BARON. As velocidades da água e os sentidos de escoamento encontrados para os tubos e, as pressões calculadas para os nós são apresentadas na Tabela 6. Na Tabela 7 é apresentada uma comparação com algumas diferentes abordagens que resolveram o problema. O melhor valor encontrado foi o custo de $\$ 419.000$, que corresponde ao ótimo global.

Na Tabela 7 são citados apenas dois autores para efeito de comparação de resultados para a rede Two loop, que usaram diferentes abordagens e o uso de simuladores hidráulicos. Surco et al. (2018a) e Ezzeldin \& Djebedjian (2020) usaram o simulador EPANET em abordagens meta-heurísticas, com PSO e WOA. Muitos outros autores também utilizaram esse problema e o melhor resultado encontrado é o mesmo apresentado nesse trabalho, que corresponde ao ótimo global para este problema.

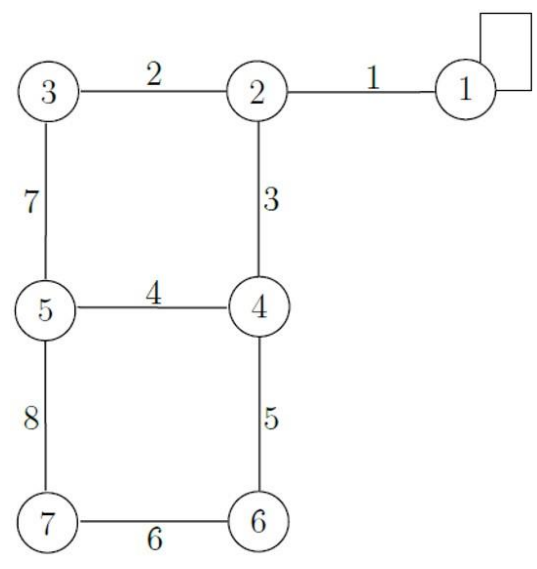

Figura 2 - Rede Two loop 
Tabela 4 - Características dos nós para a rede Two loop

\begin{tabular}{c|c|c}
\hline Nó & Elevação (m) & Demanda (m $\mathbf{3} / \mathbf{h})$ \\
\hline 1 & 210 & -1120 \\
\hline 2 & 150 & 100 \\
\hline 3 & 160 & 100 \\
\hline 4 & 155 & 120 \\
\hline 5 & 150 & 270 \\
\hline 6 & 165 & 330 \\
\hline 7 & 160 & 200 \\
\hline
\end{tabular}

Tabela 5 - Dados dos diâmetros disponíveis para a rede Two loop

\begin{tabular}{c|c|c|c}
\hline \multicolumn{2}{c|}{ Diâmetro (m) } & Diâmetro (m) & Custo (\$/m) \\
\hline 0,0254 & 2 & 0,3048 & 50 \\
\hline 0,0508 & 5 & 0,3556 & 60 \\
\hline 0,0762 & 8 & 0,4064 & 90 \\
\hline 0,1016 & 11 & 0,4572 & 130 \\
\hline 0,1524 & 16 & 0,5080 & 170 \\
\hline 0,2032 & 23 & 0,5588 & 300 \\
\hline 0,2540 & 32 & 0,6096 & 550 \\
\hline
\end{tabular}

Tabela 6 - Variáveis hidráulicas calculadas para a rede Two loop

\begin{tabular}{c|c|c|c|c}
\hline \multicolumn{1}{c}{ Tubo } & Selocidade (m/s) & $1-2$ & Nó & 1 \\
\hline 1 & 1,90 & $2-3$ & 2 & 210 \\
\hline 2 & 1,85 & $2-4$ & 3 & 53,25 \\
\hline 3 & 1,46 & $4-5$ & 4 & 30,46 \\
\hline 4 & 1,12 & $4-6$ & 5 & 43,45 \\
\hline 5 & 1,14 & $6-7$ & 6 & 33,80 \\
\hline 6 & 1,10 & $3-5$ & 3 & 30,44 \\
\hline 7 & 1,30 & $7-5$ & & 30,55 \\
\hline 8 & 0,31 & & & \\
\hline
\end{tabular}

Tabela 7 - Diâmetros e custo para a rede Two loop

\begin{tabular}{c|c|c|c}
\hline \multicolumn{1}{c}{ Tubo } & Surco et al. (2018a) & Ezzeldin \& Djebedjian (2020) & Presente trabalho \\
\hline 1 & 0,4572 & 0,4572 & 0,4572 \\
\hline 2 & 0,2540 & 0,2540 & 0,2540 \\
\hline 3 & 0,4064 & 0,4064 & 0,4064 \\
\hline 4 & 0,1016 & 0,1016 & 0,1016 \\
\hline 5 & 0,4064 & 0,4064 & 0,4064 \\
\hline 6 & 0,2540 & 0,2540 & 0,2540 \\
\hline 7 & 0,2540 & 0,2540 & 0,2540 \\
\hline 8 & 0,0254 & 0,0254 & 0,0254 \\
\hline Custo $(\$)$ & 419.000 & 419.000 & 419.000 \\
\hline
\end{tabular}

\subsection{Estudo de Caso 3}

Esse estudo de caso foi apresentado por Geem (2006) e é conhecido como RDA de GoYang, na Coréia do Sul. A Figura 3 representa sua topologia, contendo 1 reservatório, 30 tubos interligando 22 nós de consumo, 9 loops e 1 bomba hidráulica de 15,61 m no primeiro tubo, proporcionando mais autonomia para a rede. A alimentação dessa rede é feita por gravidade e o reservatório é o primeiro nó. As características dos nós e os comprimentos dos tubos são apresentados na Tabela 8. Para a rede de GoYang são disponibilizados 8 diâmetros comerciais, totalizando $8^{30}$ possíveis soluções para 0 problema (Tabela 9), em que o coeficiente adimensional de rugosidade é 100 para todos os diâmetros disponíveis. A pressão mínima é de $15 \mathrm{~m}$ para todos os nós. Para este problema, utiliza-se dois parâmetros diferentes para a equação de Hazen-Williams, que são $\alpha=10,5879$ e $\alpha=10,667$. Os outros dois parâmetros fixos são $\beta=1,852$ e $\gamma=4,871$. Utilizou-se estes dois valores para mostrar como a equação de Hazen-Williams é dependente dessa constante.

0 problema foi resolvido no ambiente GAMS com o solver SBB. Os sentidos de escoamento encontrados para os tubos e as pressões calculadas são apresentados na Tabela 10. A Tabela 11 mostra uma comparação com outras abordagens para a solução do problema publicadas na literatura. Como pode ser visto, os melhores valores encontrados foram os custos de $\$ 176.994 .561$, com $\alpha=10,5879$, e de $\$ 177.009 .557$, com $\alpha=10,667$. 
Os autores citados na comparação de resultados para a rede de GoYang fizeram uso do simulador hidráulico EPANET e diferentes abordagens foram usadas. Geem (2006) usou HS e para os parâmetros da equação de Hazen-Williams $\alpha=10,5879, \beta=1,852$ e $\gamma=4,871$. Eryiğit (2015) utilizou AIS e considerou duas situações distintas, com dois valores diferentes para o parâmetro $\alpha: \alpha=10,5879$ ou $\alpha=10,667$, na equação de Hazen-Williams. Os diferentes valores de $\alpha$ proporcionaram resultados distintos, evidenciando uma pequena sensibilidade da equação de Hazen-Williams a este parâmetro. Apesar dos valores distintos para o parâmetro $\alpha$, ao final, os resultados não mudaram muito, à exceção de um dos diâmetros.



Figura 3 - Rede de GoYang

Tabela 8 - Características dos nós e dos tubos para a rede de GoYang

\begin{tabular}{c|c|c|c|c}
\hline Nó & Elevação (m) & Demanda ( $\left.\mathbf{m}^{\mathbf{3}} / \mathbf{d i a}\right)$ & Tubo & Comprimento (m) \\
\hline 1 & 71,0 & $-2550,0$ & 1 & 165 \\
\hline 2 & 56,4 & 153,0 & 2 & 124 \\
\hline 3 & 53,8 & 70,5 & 3 & 81 \\
\hline 4 & 54,9 & 58,5 & 4 & 134 \\
\hline 5 & 56,0 & 75,0 & 5 & 135 \\
\hline 6 & 57,0 & 67,5 & 6 & 202 \\
\hline 7 & 53,9 & 63,0 & 7 & 135 \\
\hline 8 & 54,5 & 48,0 & 8 & 170 \\
\hline 9 & 57,9 & 42,0 & 9 & 113 \\
\hline 10 & 62,1 & 30,0 & 10 & 335 \\
\hline 11 & 62,8 & 42,0 & 11 & 115 \\
\hline 12 & 58,6 & 37,5 & 12 & 345 \\
\hline 13 & 59,3 & 37,5 & 13 & 114 \\
\hline 14 & 59,8 & 63,0 & 14 & 261 \\
\hline 15 & 59,2 & 445,5 & 15 & 72 \\
\hline 16 & 53,6 & 108,0 & 16 & 373 \\
\hline 17 & 54,8 & 79,5 & 17 & 98 \\
\hline 18 & 55,1 & 55,5 & 18 & 110 \\
\hline 19 & 54,2 & 118,5 & 19 & 98 \\
\hline 20 & 54,5 & 124,5 & 20 & 246 \\
\hline 21 & 62,9 & 31,5 & 21 & 174 \\
\hline 22 & 61,8 & 799,5 & 22 & 102 \\
\hline & & & 23 & 92 \\
\hline & & & 24 & 100 \\
\hline & & & 25 & 90 \\
\hline & & & 26 & 90 \\
\hline & & & 27 & 28 \\
\hline
\end{tabular}

Tabela 9 - Dados dos diâmetros disponíveis para a rede GoYang

\begin{tabular}{c|c|c|c}
\hline \multicolumn{2}{c|}{ Diâmetro (m) } & Diâmetro (m) & Custo (\$/m) \\
\hline 0,080 & 37.890 & 0,200 & 47.624 \\
\hline 0,100 & 38.933 & 0,250 & 54.125 \\
\hline 0,125 & 40.563 & 0,300 & 62.109 \\
\hline 0,150 & 42.554 & 0,350 & 71.524 \\
\hline
\end{tabular}


Tabela 10 - Variáveis hidráulicas calculadas para a rede GoYang

\begin{tabular}{|c|c|c|c|c|c|}
\hline Tubo & $\begin{array}{l}\text { Sentido de escoamento } \\
\qquad \alpha=10,5879\end{array}$ & $\begin{array}{l}\text { Sentido de escoamento } \\
\qquad \alpha=10,667\end{array}$ & Nó & $\begin{array}{l}\text { Pressão (m) } \\
\alpha=10,5879\end{array}$ & $\begin{array}{c}\text { Pressão (m) } \\
\alpha=10,667\end{array}$ \\
\hline 1 & $1-2$ & $1-2$ & 1 & 71 & 71 \\
\hline 2 & $2-3$ & $2-3$ & 2 & 24,99 & 24,95 \\
\hline 3 & $3-4$ & $3-4$ & 3 & 26,30 & 26,25 \\
\hline 4 & $4-5$ & $4-5$ & 4 & 24,07 & 24,02 \\
\hline 5 & $5-6$ & $5-6$ & 5 & 22,75 & 22,37 \\
\hline 6 & $6-12$ & $6-12$ & 6 & 20,66 & 20,57 \\
\hline 7 & $12-15$ & $12-15$ & 7 & 25,20 & 25,14 \\
\hline 8 & $2-22$ & $2-22$ & 8 & 24,35 & 24,29 \\
\hline 9 & $2-21$ & $2-21$ & 9 & 19,98 & 19,90 \\
\hline 10 & $21-22$ & $21-22$ & 10 & 15,42 & 15,07 \\
\hline 11 & $20-22$ & $20-22$ & 11 & 15,05 & 15,04 \\
\hline 12 & $19-20$ & $19-20$ & 12 & 18,16 & 17,79 \\
\hline 13 & $2-19$ & $2-19$ & 13 & 17,38 & 17,15 \\
\hline 14 & $17-19$ & $17-19$ & 14 & 15,30 & 15,00 \\
\hline 15 & $3-16$ & $3-16$ & 15 & 15,46 & 15,14 \\
\hline 16 & $16-17$ & $16-17$ & 16 & 25,69 & 25,63 \\
\hline 17 & $18-17$ & $18-17$ & 17 & 23,85 & 23,79 \\
\hline 18 & $7-18$ & $7-18$ & 18 & 23,56 & 23,50 \\
\hline 19 & $3-7$ & $3-7$ & 19 & 24,35 & 24,28 \\
\hline 20 & $7-8$ & $7-8$ & 20 & 23,42 & 23,36 \\
\hline 21 & $4-8$ & $4-8$ & 21 & 16,09 & 16,03 \\
\hline 22 & $8-9$ & $8-9$ & 22 & 15,88 & 15,81 \\
\hline 23 & $5-11$ & $5-11$ & & & \\
\hline 24 & $11-10$ & $11-10$ & & & \\
\hline 25 & $6-10$ & $6-10$ & & & \\
\hline 26 & $9-6$ & $9-6$ & & & \\
\hline 27 & $10-13$ & $10-13$ & & & \\
\hline 28 & $12-13$ & $13-12$ & & & \\
\hline 29 & $13-14$ & $13-14$ & & & \\
\hline 30 & 14-15 & $14-15$ & & & \\
\hline
\end{tabular}

Tabela 11 - Diâmetros e custos para a rede GoYang

\begin{tabular}{|c|c|c|c|c|c|}
\hline Tubo & $\begin{array}{c}\text { Geem (2006) } \\
\alpha=10,5879\end{array}$ & $\begin{array}{c}\text { Eryiğit (2015) } \\
\alpha=10,5879\end{array}$ & $\begin{array}{l}\text { Presente trabalho } \\
\alpha=10,5879\end{array}$ & $\begin{array}{l}\text { Eryiğit (2015) } \\
\quad \alpha=10,667\end{array}$ & $\begin{array}{c}\text { Presente trabalho } \\
\alpha=10,667\end{array}$ \\
\hline 1 & 0,150 & 0,150 & 0,150 & 0,150 & 0,150 \\
\hline 2 & 0,150 & 0,150 & 0,150 & 0,150 & 0,150 \\
\hline 3 & 0,125 & 0,125 & 0,125 & 0,125 & 0,125 \\
\hline 4 & 0,150 & 0,150 & 0,150 & 0,125 & 0,125 \\
\hline 5 & 0,100 & 0,100 & 0,100 & 0,100 & 0,100 \\
\hline 6 & 0,100 & 0,100 & 0,100 & 0,080 & 0,080 \\
\hline 7 & 0,080 & 0,080 & 0,080 & 0,080 & 0,080 \\
\hline 8 & 0,100 & 0,080 & 0,080 & 0,080 & 0,080 \\
\hline 9 & 0,080 & 0,080 & 0,080 & 0,080 & 0,080 \\
\hline 10 & 0,080 & 0,080 & 0,080 & 0,080 & 0,080 \\
\hline 11 & 0,080 & 0,080 & 0,080 & 0,080 & 0,080 \\
\hline 12 & 0,080 & 0,080 & 0,080 & 0,080 & 0,080 \\
\hline 13 & 0,080 & 0,080 & 0,080 & 0,080 & 0,080 \\
\hline 14 & 0,080 & 0,080 & 0,080 & 0,080 & 0,080 \\
\hline 15 & 0,080 & 0,080 & 0,080 & 0,080 & 0,080 \\
\hline 16 & 0,080 & 0,080 & 0,080 & 0,080 & 0,080 \\
\hline 17 & 0,080 & 0,080 & 0,080 & 0,080 & 0,080 \\
\hline 18 & 0,080 & 0,080 & 0,080 & 0,080 & 0,080 \\
\hline 19 & 0,080 & 0,080 & 0,080 & 0,080 & 0,080 \\
\hline 20 & 0,080 & 0,080 & 0,080 & 0,080 & 0,080 \\
\hline 21 & 0,080 & 0,080 & 0,080 & 0,080 & 0,080 \\
\hline 22 & 0,080 & 0,080 & 0,080 & 0,080 & 0,080 \\
\hline 23 & 0,080 & 0,080 & 0,080 & 0,100 & 0,100 \\
\hline 24 & 0,080 & 0,080 & 0,080 & 0,080 & 0,080 \\
\hline 25 & 0,080 & 0,080 & 0,080 & 0,080 & 0,080 \\
\hline 26 & 0,080 & 0,080 & 0,080 & 0,080 & 0,080 \\
\hline 27 & 0,080 & 0,080 & 0,080 & 0,100 & 0,100 \\
\hline 28 & 0,080 & 0,080 & 0,080 & 0,080 & 0,080 \\
\hline 29 & 0,080 & 0,080 & 0,080 & 0,080 & 0,080 \\
\hline 30 & 0,080 & 0,080 & 0,080 & 0,080 & 0,080 \\
\hline Custo (\$) & 177.135 .800 & 176.994 .561 & 176.994 .561 & 177.009 .557 & 177.009 .557 \\
\hline
\end{tabular}


O solver BARON (Branch-And-Reduce Optimization Navigator), no ambiente GAMS, é um solver de otimização global para a solução de problemas com formulação de programação não linear (NLP) e programação não linear mista com variáveis inteiras (MINLP). 0 solver implementa técnicas de otimização global determinísticas com algoritmos do tipo Branch and Bound que garantem otimalidade global dentro de imposições de finitos limites superiores e inferiores para as expressões (igualdades e desigualdades) no modelo a ser resolvido. No entanto, para problemas de grande porte, como o caso de estudo 3, o solver começa a ter dificuldades em fechar o gap formado entre os limites inferior e superior para essas expressões. Por isso, foi utilizado o solver SBB (Spatial Branch and Boud) que é também um solver para problemas com formulação MINLP e é baseado em uma combinação do método Branch and Bound e alguns solvers padrão para a solução de problemas NLP com boas estimativas iniciais.

\section{CONCLUSÕES}

No presente artigo, foi desenvolvido um modelo de otimização com formulação MINLP para o projeto ótimo de RDA pressurizadas e em loop, considerando os sentidos de escoamento da água desconhecidos nos tubos, simultaneamente com os diâmetros dos tubos e com o custo total de instalação da rede. 0 modelo foi reformulado utilizando programação disjuntiva e estratégias para tratar as não linearidades nas equações foram aplicadas para evitar complexidades desnecessárias e permitiram a utilização de técnicas de otimização global em ambiente GAMS. 0 sistema não linear formado pelas equações hidráulicas pertencentes ao problema foi resolvido sem a necessidade de software auxiliar para o cálculo das velocidades e pressões.

O modelo de otimização permite obter o custo mínimo de instalação da rede, que depende dos diâmetros comerciais dos tubos. Três estudos de caso da literatura foram usados na aplicação e excelentes resultados foram encontrados, como pode ser visto na comparação com trabalhos existentes. A maioria dos casos (83\% dos artigos publicados no assunto) consideram os sentidos de escoamento fixos. Na rede de GoYang, evidenciou-se a influência dos parâmetros da equação de HazenWilliams no processo de otimização.

A síntese de RDA é um problema complexo, devido à resolução de um sistema de equações não lineares, com algumas variáveis discretas. Quando os sentidos de escoamento são considerados fixos, a priori, o problema se limita às soluções fornecidas para a estrutura considerada. Quando os sentidos de escoamento são considerados variáveis do problema, a complexidade no projeto da rede é aumentada. Entretanto as aplicações são mais realistas, podendo trabalhar em diferentes situações e estruturas.

\section{AGRADECIMENTOS}

Os autores agradecem o apoio financeiro do Conselho Nacional para o Desenvolvimento Científico e Tecnológico - CNPq, por meio dos projetos 311807/2018-6 e 428650/2018-0.

\section{NOMENCLATURA}

$C_{i, j}$ Coeficiente de rugosidade de Hazen-Williams no trecho $i-j$ [adm]

$C_{T}$ Custo total de instalação da rede [\$]

Custo $\left(D_{k}\right)$ Custo por comprimento do tubo com diâmetro disponível $D_{k}[\$ / \mathrm{m}]$

Custo $\left(x_{i, j}\right)$ Custo por comprimento do tubo no trecho $i-j$ com diâmetro $x_{i, j}[\$ / \mathrm{m}]$

$\mathcal{D}$ Conjunto dos diâmetros comerciais disponíveis $(k)$

$D_{k}$ Diâmetro comercial disponível $k[\mathrm{~m}]$

$d_{i}$ Demanda de água para o nó $i$ [volume/tempo]

$\mathcal{E}_{i, j}$ Existência de uma ligação entre o nó $i$ e o nó $j(i-j)$

$E p_{i, j}$ Altura Manométrica, ou Elevação de Pressão, gerada por uma Bomba hidráulica no trecho

$i-j[\mathrm{~m}]$

$E p_{i, j}^{1}$ Igual a $E p_{i, j}$ se a água flui do nó $i$ para o nó $j$, zero caso contrário

$E p_{i, j}^{2}$ Igual a $E p_{i, j}$ se a água flui do nó $j$ para o nó $i$, zero caso contrário

$E p_{i, j}^{m i n}$ Valor mínimo para a bomba hidráulica no trecho $i-j[\mathrm{~m}]$

$E p_{i, j}^{\max }$ Valor máximo para a bomba hidráulica no trecho $i-j[\mathrm{~m}]$

$h_{i}$ Elevação do nó $i$ [m]

$i, j$ Nó de demanda

$k$ Diâmetro disponível

$L_{i, j}$ Comprimento do trecho $i-j[\mathrm{~m}]$ 


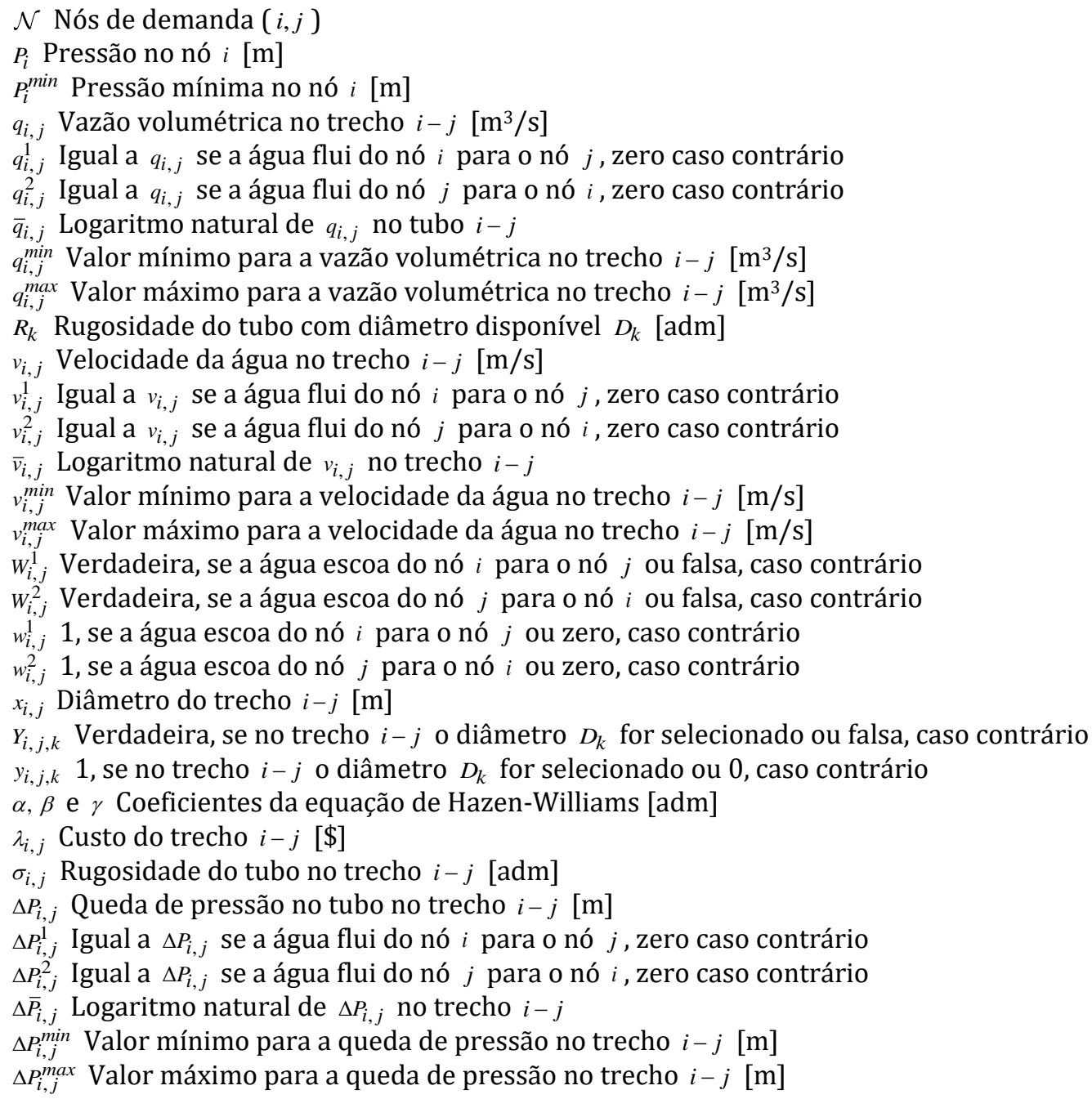

\section{REFERÊNCIAS}

Alperovits, E., \& Shamir, U. (1977). Design of optimal water distribution systems. Water Resources Research, 13(6), 885-900. http://dx.doi.org/10.1029/WR013i006p00885

Avila-Melgar, E. Y., Cruz-Chávez, M. A., \& Martinez-Bahena, B. (2017). General methodology for using epanet as an optimization element in evolutionary algorithms in a grid computing environment for water distribution network design. Water Supply, 17(1), 39-51. http://dx.doi.org/10.2166/ws.2016.101

Balas, E. (2018). Disjunctive programming (1st ed.). Switzerland: Springer. http://dx.doi.org/10.1007/978-3030-00148-3.

Bi, W., Dandy, G., \& Maier, H. (2015). Improved genetic algorithm optimization of water distribution system design by incorporating domain knowledge. Environmental Modelling \& Software, 69, 370-381. http://dx.doi.org/10.1016/j.envsoft.2014.09.010

Bolognesi, A., Bragalli, C., Marchi, A., \& Artina, S. (2010). Genetic heritage evolution by stochastic transmission in the optimal design of water distribution networks. Advances in Engineering Software, 41(5), 792-801. http://dx.doi.org/10.1016/j.advengsoft.2009.12.020

Bragalli, C., D’Ambrosio, C., Lee, J., Lodi, A., \& Toth, P. (2006). An MINLP solution method for a water network problem. In Algorithms - ESA 2006, 14th Annual European Symposium (pp. 696-707). Berlin: Springer. http://dx.doi.org/10.1007/11841036_62.

Bragalli, C., D’Ambrosio, C., Lee, J., Lodi, A., \& Toth, P. (2008). Water network design by MINLP (Rep. No. RC24495, pp. 1-17). Yorktown Heights: IBM Research.

Bragalli, C., D'Ambrosio, C., Lee, J., Lodi, A., \& Toth, P. (2012). On the optimal design of water distribution networks: a practical MINLP approach. Optimization and Engineering, 13(2), 219-246.

http://dx.doi.org/10.1007/s11081-011-9141-7 
Carvalho, P. S. O. (2007). EficientE: um método de dimensionamento econômico para redes de distribuição de água (Dissertação de mestrado). Universidade Federal da Paraíba, João Pessoa.

Cassiolato, G. H. B., Carvalho, E. P., Caballero, J. A., \& Ravagnani, M. A. S. S. (2019). Water distribution networks optimization using generalized disjunctive programming. Chemical Engineering Transactions, 76, 547-552. Recuperado em 10 de agosto de 2021, de https://www.cetjournal.it/index.php/cet/article/view/CET1976092

Cassiolato, G. H. B., Carvalho, E. P., Caballero, J. A., \& Ravagnani, M. A. S. S. (2021). Optimization of water distribution networks using a deterministic approach. Engineering Optimization, 53(1), 107-124. http://dx.doi.org/10.1080/0305215X.2019.1702980

D’Ambrosio, C., Lodi, A., Wiese, S., \& Bragalli, C. (2015). Mathematical programming techniques in water network optimization. European Journal of Operational Research, 243(3), 774-788. http://dx.doi.org/10.1016/j.ejor.2014.12.039

Eiger, G., Shamir, U., \& Ben-Tal, A. (1994). Optimal design of water distribution networks. Water Resources Research, 30(9), 2637-2646. http://dx.doi.org/10.1029/94WR00623

Eryiğit, M. (2015). Cost optimization of water distribution networks by using artificial immune systems. Journal of Water Supply: Research \& Technology - Aqua, 64(1), 47-63. http://dx.doi.org/10.2166/aqua.2014.031

Ezzeldin, R., Djebedjian, B., \& Saafan, T. (2014). Integer discrete particle swarm optimization of water distribution networks. Journal of Pipeline Systems Engineering and Practice, 5(1), 04013013. http://dx.doi.org/10.1061/(ASCE)PS.1949-1204.0000154

Ezzeldin, R. M., \& Djebedjian, B. (2020). Optimal design of water distribution networks using whale optimization algorithm. Urban Water Journal, 17(1), 14-22. http://dx.doi.org/10.1080/1573062X.2020.1734635

Fallah, H., Ghazanfari, S., Suribabu, C. R., \& Rashedi, E. (2021). Optimal pipe dimensioning in water distribution networks using gravitational search algorithm. ISH Journal of Hydraulic Engineering, 27(Supl. 1), 242-255. http://dx.doi.org/10.1080/09715010.2019.1624630

Geem, Z. W. (2006). Optimal cost design of water distribution networks using harmony search. Engineering Optimization, 38(3), 259-277. http://dx.doi.org/10.1080/03052150500467430

Geem, Z. W. (2009). Particle-swarm harmony search for water network design. Engineering Optimization, 41(4), 297-311. http://dx.doi.org/10.1080/03052150802449227

Goulter, I. C., Lussier, B. M., \& Morgan, D. R. (1986). Implications of head loss path choice in the optimization of water distribution networks. Water Resources Research, 22(5), 819-822. http://dx.doi.org/10.1029/WR022i005p00819

Grossmann, I. E., \& Lee, S. (2003). Generalized convex disjunctive programming: nonlinear convex hull relaxation. Computational Optimization and Applications, 26(1), 83-100. http://dx.doi.org/10.1023/A:1025154322278

Hansen, C. T., Madsen, K., \& Nielsen, H. B. (1991). Optimization of pipe networks. Mathematical Programming, 52(1-3), 45-58. http://dx.doi.org/10.1007/BF01582879

Kadu, M. S., Gupta, R., \& Bhave, P. R. (2008). Optimal design of water networks using a modified genetic algorithm with reduction in search space. Journal of Water Resources Planning and Management, 134(2), 147-160. http://dx.doi.org/10.1061/(ASCE)0733-9496(2008)134:2(147)

Kessler, A., \& Shamir, U. (1989). Analysis of the linear programming gradient method for optimal design of water supply networks. Water Resources Research, 25(7), 1469-1480. http://dx.doi.org/10.1029/WR025i007p01469

Mala-Jetmarova, H., Sultanova, N., \& Savic, D. (2018). Lost in optimisation of water distribution systems? a literature review of system design. Water, 10(3), 307. http://dx.doi.org/10.3390/w10030307

Morsi, A., Geissler, B., \& Martin, A. (2012). Mixed integer optimization of water supply networks. In A. Martin, K. Klamroth, J. Lang, G. Leugering, A. Morsi, M. Oberlack, M. Ostrowski \& R. Rosen (Orgs.), Mathematical optimization of water networks (Vol. 162, pp. 35-54). Berlin: Springer. http://dx.doi.org/10.1007/978-3-0348-0436-3_3.

Palod, N., Prasad, V., \& Khare, R. (2020). Non-parametric optimization technique for water distribution in pipe networks. Water Supply, 20(8), 3068-3082. http://dx.doi.org/10.2166/ws.2020.200

Rao, C. J., Jothiprakash, V., \& Eldho, T. I. (2017). Design of a pipe network using the finite-element method coupled with particle-swarm optimization. Journal of Pipeline Systems Engineering and Practice, 8(4), 04017019. http://dx.doi.org/10.1061/(ASCE)PS.1949-1204.0000282

Reca, J., \& Martínez, J. (2006). Genetic algorithms for the design of looped irrigation water distribution networks. Water Resources Research, 42(5), 1-9. http://dx.doi.org/10.1029/2005WR004383

Reca, J., Martínez, J., \& López, R. (2017). A hybrid water distribution networks design optimization method based on a search space reduction approach and a genetic algorithm. Water, 9(11), 845. http://dx.doi.org/10.3390/w9110845 
Sarbu, I. (1997). Optimal design of water distribution networks. Journal of Hydraulic Research, 35(1), 63-79. http://dx.doi.org/10.1080/00221689709498644

Savic, D. A., \& Walters, G. A. (1997). Genetic algorithms for least-cost design of water distribution networks. Journal of Water Resources Planning and Management, 123(2), 67-77. http://dx.doi.org/10.1061/(ASCE)07339496(1997)123:2(67)

Shamir, U. Y., \& Howard, C. D. D. (1968). Water distribution systems analysis. Journal of the Hydraulics Division, 94(1), 219-234. http://dx.doi.org/10.1061/JYCEAJ.0001747

Shende, S., \& Chau, K. W. (2019). Design of water distribution systems using an intelligent simple benchmarking algorithm with respect to cost optimization and computational efficiency. Water Supply, 19(7), 1892-1898. http://dx.doi.org/10.2166/ws.2019.065

Surco, D. F., Macowski, D. H., Coral, J. G. L., Cardoso, F. A. R., Vecchi, T. P. B., \& Ravagnani, M. A. S. S. (2019). Multiswarm optimizer applied in water distribution networks. Desalination and Water Treatment, 161, 1-13. http://dx.doi.org/10.5004/dwt.2019.24146

Surco, D. F., Vecchi, T. P. B., \& Ravagnani, M. A. S. S. (2018a). Optimization of water distribution networks using a modified particle swarm optimization algorithm. Water Supply, 18(2), 660-678. http://dx.doi.org/10.2166/ws.2017.148

Surco, D. F., Vecchi, T. P. B., \& Ravagnani, M. A. S. S. (2018b). Rehabilitation of water distribution networks using particle swarm optimization. Desalination and Water Treatment, 106, 312-329. http://dx.doi.org/10.5004/dwt.2018.22101

Surco, D. F., Macowski, D. H., Cardoso, F. A. R., Vecchi, T. P. B., \& Ravagnani, M. A. S. S. (2021). Multi-objective optimization of water distribution networks using PSO. Desalination and Water Treatment, 218, 18-31. http://dx.doi.org/10.5004/dwt.2021.26944

Suribabu, C., \& Neelakanta, T. (2014). Optimal upgradation and expansion of existing water distribution networks using differential evolution algorithm. Asian Journal of Applied Sciences, 7(6), 375-390. http://dx.doi.org/10.3923/ajaps.2014.375.390

Suribabu, C. R. (2010). Differential evolution algorithm for optimal design of water distribution networks. Journal of Hydroinformatics, 12(1), 66-82. http://dx.doi.org/10.2166/hydro.2010.014

Suribabu, C. R., \& Neelakantan, T. R. (2006). Design of water distribution networks using particle swarm optimization. Urban Water Journal, 3(2), 111-120. http://dx.doi.org/10.1080/15730620600855928

Vasan, A., \& Simonovic, S. P. (2010). Optimization of water distribution network design using differential evolution. Journal of Water Resources Planning and Management, 136(2), 279-287. http://dx.doi.org/10.1061/(ASCE)0733-9496(2010)136:2(279)

Watanatada, T. (1973). Least-cost design of water distribution systems. Journal of the Hydraulics Division, 99(9), 1497-1513. http://dx.doi.org/10.1061/JYCEAJ.0003742

Contribuições dos autores:

Gustavo Henrique Batista Cassiolato: desenvolvimento do modelo matemático e suas reformulações, estudos de caso e redação do texto.

Esdras Penêdo de Carvalho: desenvolvimento do modelo matemático e suas reformulações, estudos de caso e redação do texto.

Mauro Antonio da Silva Sá Ravagnani: desenvolvimento do modelo matemático e suas reformulações, estudos de caso e redação do texto. 